\title{
Common-Neutral-Type AC/DC/AC Topologies with PFC Pre-regulator
}

\author{
Wen-Jung Ho, Mu-Shen Lin, Wu-Shiung Feng \\ Department of Electrical Engineering \\ National Taiwan University \\ Taipei 10617 , Taiwan
}

\begin{abstract}
In this paper new topologies of single-phase PFC-pre-staged $A C / D C / A C$ converter are presented. By introducing the common-neutral connection to simplify a conventional connection of $A C / D C / A C$ structure, they derive new topologies but keep good performance, output safety-code conformed and provide extra advantages - fewer power devices and lower conduction losses.
\end{abstract}

\section{Introduction}

With the utility systems being fluctuated and contaminated easily, the demand for $\mathrm{AC} / \mathrm{DC} / \mathrm{AC}$ converter with input power factor correction (PFC) is growing rapidly because it can serve as the main power stage of uninterruptible power supply (UPS), AC cycloconverter, and $\mathrm{AC}$ line conditioner etc. [1 4].

A PFC-pre-staged AC/DC/AC converter with common-neutral connection consists of a pre-staged PFC and a post-staged PWM inverter. For example, connecting a two-switch voltage-doubler PFC and half-bridge inverter via common-neutral connection (bold-line) can built a frugal structure as shown in Fig. 1 [1]. This $\mathrm{AC} / \mathrm{DC} / \mathrm{AC}$ structure can satisfy the demands of cost reduction and compliance of safety-code. Although its performance is not good enough because it has to use non-optimal bipolar PWM switching scheme for $\mathrm{PFC}$ and inverter.

PFC and inverter have been the source of interest in the literature for many years, yet very little is discussed about the relationships of connection. Hence, this paper explores new topologies of $\mathrm{AC} / \mathrm{DC} / \mathrm{AC}$ converter with common-neutral connection and their superior performance.

\section{II.The Proposed AC/DC/AC Topologies}

\section{A. An Economical $A C / D C / A C$ \\ Topologies with Common-Neutral \\ Connection}

Connecting a single-switch voltage-doubler PFC and half-bridge inverter via common-neutral connection of bold-line can built an improvement structure as shown in Fig. 2. Although it has half-bridge inverter 
with non-optimal bipolar PWM switching scheme, the PFC employs unipolar-voltage switching scheme which yields lower input current distortion and less EMI [2].

Switches $T_{a}$ are controlled to shape the input current to be sinusoidal for PFC function; switches $T_{1} \sim T_{2}$ are operated in a PWM fashion to generate output voltage for inverter function. When input voltage is positive, switch $T_{a}$ turns on, $I_{i}$ goes forward to energize $L i$, then off boosts input via $D_{1}$ to charge upper DC capacitor, shaping the input current to be sinusoidal in phase with input voltage simultaneously. In the negative cycle, also do the same job but charge the other DC capacitor.

\section{B. H-bridge AC/DC/AC Topologies with Common-Neutral Connection}

Fig. 3 shows a conventional connection of AC/DC/AC structure which consists of a 2-switch H-bridge $\mathrm{PFC}$, full-bridge inverter and isolation transformer, including all dash-line components (pseudo-switch open). It is of a performance-oriented structure because the PFC employs unipolarvoltage switching scheme which yields lower input current distortion and less EMI, and the full-bridge inverter is more capable of undertaking reactive and heavy load [3]. However, this configuration has more power transistors and diodes than Fig. 1 and Fig. 2, and especially it has to add a bulk isolation transformer to the output. Because the neutral-ground voltage $V_{A G}$ is of PWM-related waveforms during inverter transistors $T_{1} \sim T_{4}$ switching. If without the transformer, the new output terminal, denoted by $o / p^{\prime}$, cannot connect with the ground $G$ to comply with safety code [4]. Fig. 4 shows the simulated voltage waveforms we interest. We can find that $V_{A G}$ is a quasi-sinusoidal waveform carrying with saw-toothed PWM, and its amplitude is around half the inverter output $V_{A D}$. and dangerous to be touched. Hence, the bulk transformer is an inevitable device to separate $V_{A G}$ and connect the second side of it to the ground.

The proposed topology is also an improvement of structure, and it satisfies both sides -- the demands of performance and economy. In Fig. 3 if we connect the node $A$ with node $N$ (pseudo-switch closed) -- commonneutral connection -- we can save all the dash-line components consisting of output transformer, $D_{1}$ and $D_{2}$. Because in this way $V_{A G}=V_{N G}$, the new output $o / p^{\prime}$ can keep the same neutral-ground relationship as the input network just like Fig. 1 and Fig. 2. Usually the utility neutral-ground $V_{N G}$ is under few volts -neutral earthing through the input network -- for safely touching it [4]. Moreover, $D_{1}$ shunts with $T_{i}$ 's built-in diode and $D_{2}$ shunts with $T_{2}$ 's built-in diode. Thus both $D_{1}$ and $D_{2}$ can be omitted for redundant situation.

Because all the active components $T_{a} \sim T_{b}$ and $T_{i \sim T_{4}}$ are unchanged, the proposed structure can employ the same driving and control circuits as the original conventional connection one. Hence, switches $T_{a}$ and $T_{b}$ are controlled to shape the input current to be sinusoidal for PFC function; switches $T_{1} \sim T_{4}$ are operated in a PWM fashion to generate output voltage for inverter function. When input voltage is positive, switch $T_{b}$ turns on, $I_{i}$ goes via $T 2$ 's built-in diode (instead of $D_{2}$ ) to energize $L i$, then off 
boosts input to charge DC link capacitor, shaping the input current to be sinusoidal in phase with input voltage simultaneously. In the negative cycle, switch $T a$ with the counterpart components will also do the same job [2,3]. Fig. 5(a) and 5(b) show the test results of similar input waveforms of the conventional and proposed connection respectively.

In the positive cycle, Ion carries $I_{i}$ while $T b$ closed, and $I_{\text {off }}$ carries $I_{i}$ while $T_{b}$ open. Meanwhile, if the inverter switches $T_{I} \sim T_{4}$ work on synchronous PWM action as usual, $I_{o}$ will go through $T_{3}$ and $T_{2}$, thus the switch $T_{2}$ 's current $I_{T 2}=I_{0}-I_{o n}$ when $T_{b}$ closed $\left(I_{T 2}=I_{o}\right.$ when $T_{b}$ open) as indicated in Fig. 3. Comparably, in the topology of conventional connection, $I_{T 2}=I_{o}$ whenever $T_{b}$ is closed or open. We know the conduction losses of $T_{2}$ depend on the product of transistor voltage and current ( $P_{\text {cond loss }}=V_{T 2}$ • $I_{T 2}$ ). Therefore the proposed topology has less conduction losses because of smaller average transistor current $I_{T 2}$ when compared with the conventional connection. In the negative cycle, $T_{I}$ will have the analogous results.

\section{C. $3 \phi$ input $/ 1 \phi$ output $A C / D C / A C$ Topologies with Common-Neutral Connection}

Fig. $6 \sim$ Fig. 8 shows the topologies of 3 phase input / singlephase output, which can reduce the value of input current and the DC-bus capacitor, and many other beneficial points as well. Fig. 6 employs twoswitch voltage-doubler PFC as the input and half-bridge inverter as the output via common-neutral connection (boldline) can built a frugal structure. Fig. 7 employs single-switch voltage-doubler PFC as input and half-bridge inverter as the output via common-neutral connection of bold-line can built an improvement structure. Fig. 8 employs the proposed topology of single-phase common-neutral-type AC/DC/AC as the input (pseudo-switch closed) that is also an improvement of structure, and it satisfies both sides -- the demands of performance and economy.

\section{Test Results}

To verify the feasibility of the proposed AC/DC/AC converter, a $1 \mathrm{KW}$ prototype with 2-switch H-bridge PFC and full-bridge inverter was implemented, and then converted it to the proposed configuration and still worked well. The PFC operated around $20 \mathrm{kHz}$, and inverter operated around $10 \mathrm{kHz}$ with $L i=2.2 \mathrm{mH}, L o=2.2 \mathrm{mH}$, and Co $=10 u$ F. Fig. 5(a) and 5(b) show the test results of similar input waveforms of the conventional and proposed connection respectively. And, $I T 2=4.0 \mathrm{~A}$ $\mathrm{rms} \quad\left(I_{T 2}=5.2 \mathrm{~A} \mathrm{rms}\right.$ for conventional connection), i.e. the new topology shows lower conduction losses.

\section{Conclusions}

The new PFC-pre-staged AC/DC/AC topologies with commonneutral connection has been presented. They provides good performance, safety-code conformed, few power devices, and low conduction losses as well. 


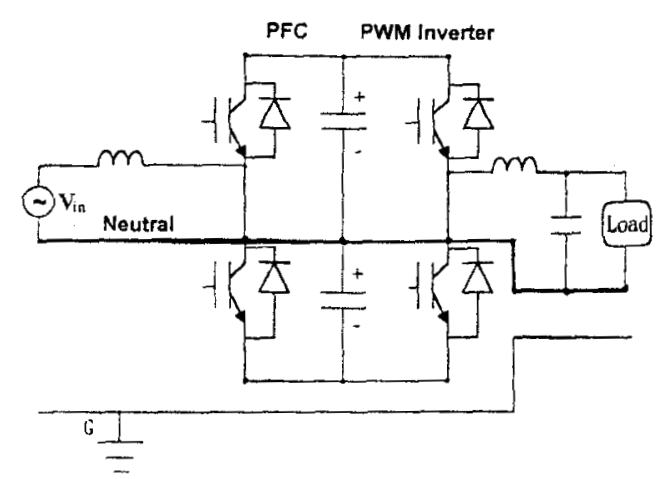

Fig. 1: An AC/DC/AC topology with common-neutral connection.

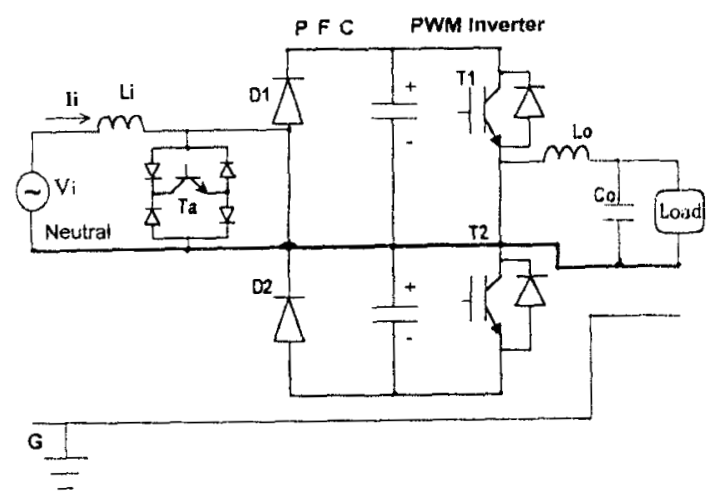

Fig. 2: The proposed AC/DC/AC topology with common-neutral connection.

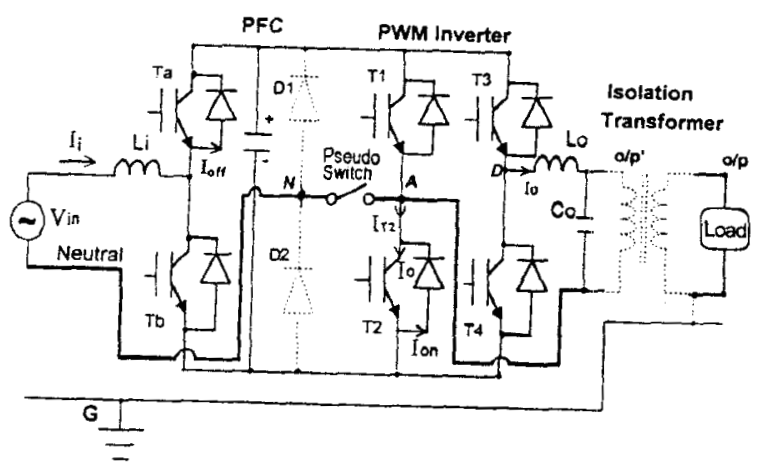

Fig. 3: A performance-oriented $\mathrm{AC} / \mathrm{DC} / \mathrm{AC}$ topology 1) pseudo-switch open -conventional connection, including dash-line devices. 2) pseudo-switch closed -- the proposed common-neutral structure, excluding dash-line devices $\left(D_{1}, D_{2}, \&\right.$ o/p transformer), the load conneted to $o / p^{\prime}$.

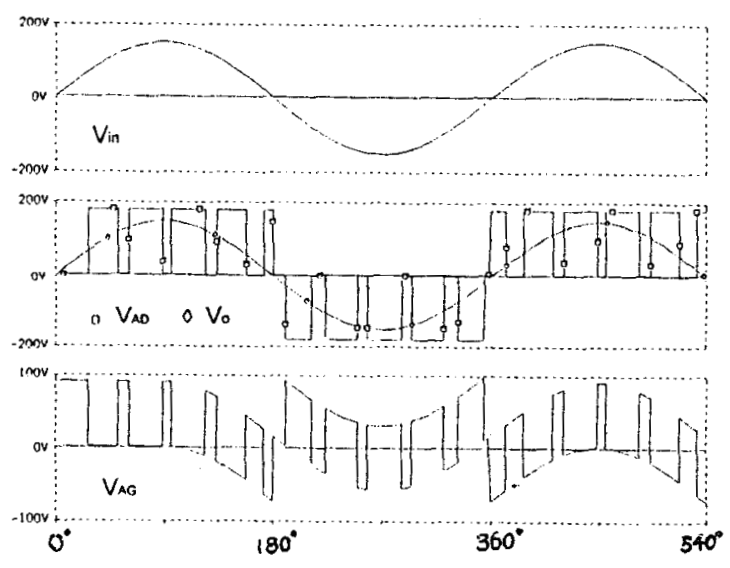

Fig. 4: Simulated waveforms of Fig. 3, including $V_{i n}, V_{A D}, V_{o}$ and $V_{A G}$. 


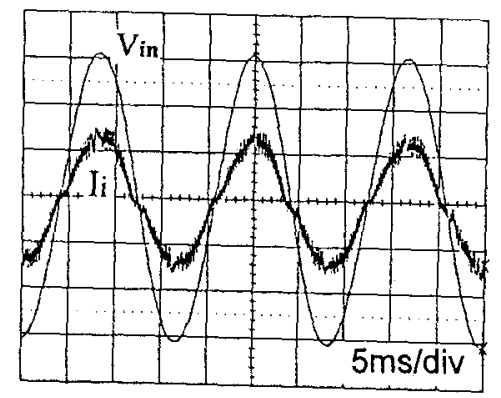

(a)

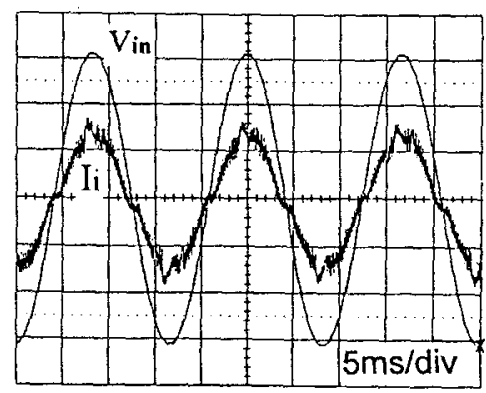

(b)

Fig. 5: (a) Input waveforms of conventional connection structure, and 5(b) Input waveforms of the proposed structure, $V_{\text {in }}(50 \mathrm{~V} / \mathrm{div})$ and $I_{i}$ (5A/div).

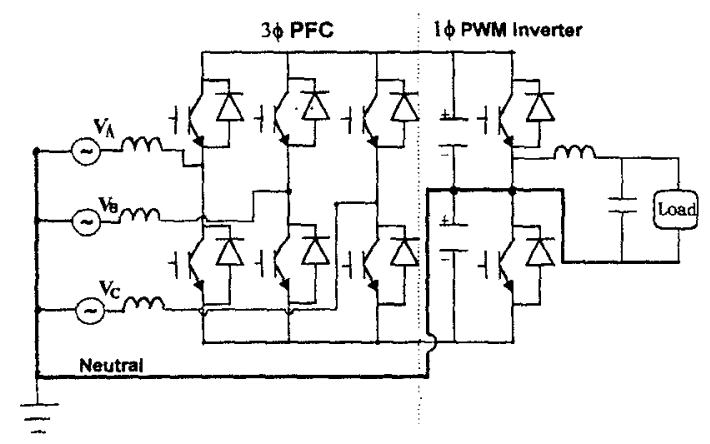

Fig. 6: $3 \phi$ input / $1 \phi$ output AC/DC/AC topologies with common-neutral connection using two-switch voltage-doubler PFC and halfbridge inverter

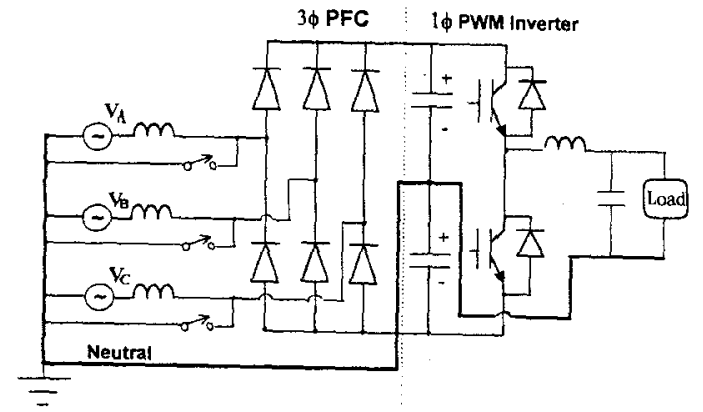

Fig. 7: $3 \phi$ input / $1 \phi$ output $\mathrm{AC} / \mathrm{DC} / \mathrm{AC}$ topology with common-neutral connection using single-switch voltage-doubler PFC and halfbridge inverter

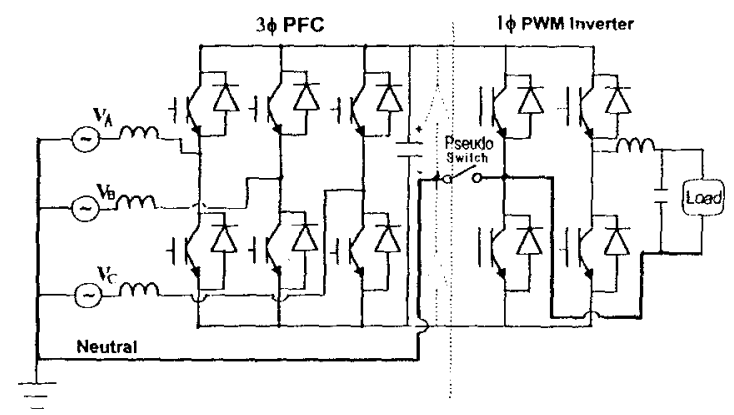

Fig. 8: $3 \phi$ input / $1 \phi$ output AC/DC/AC topology with common-neutral connection using H-bridge $\mathrm{AC} / \mathrm{DC} / \mathrm{AC}$ topology 


\section{References:}

[1] Yuasa Corporation, Osaka, Japan, " Development of UPS using new type of circuits," IEEE INTELEC'94, Conf. Proceedings, pp. 635-642.

[2] John C. Salmon, "Circuit topologies for single-phase voltage-doubler boost rectifiers, "IEEE Trans. Power Electronics, Vol. 8, No. 4, pp. 521-529, Oct. 1993.

[3] I. Kubo, Y. Ozawa, R. Nakatsuka, A. Shimizu, "A fully digital controlled UPS

using IGBT's," IEEE IAS'91, Conf. Proceedings, pp 1042-1046.

[4] Kimmo Dahl, IVO Engineering, "Different Methods of UPS Neutral and Protective Earthing and Their Impact on Human Safety and System Disturbances," IEEE INTELEC'91, Conf. Proceedings, pp. 443-448. 